\title{
Carbocyclic Carbene Ligands Derived from Aromatic Nitrones: Formation and Catalytic Activity of Their Pd (II) Complexes
}

\author{
Qingwei Yao,* Mark Zabawa, Joyce Woo, and Chong Zheng \\ Department of Chemistry and Biochemistry, The Michael Faraday Laboratories, \\ Northern Illinois University, DeKalb, Illinois 60115
}

General

Synthesis of Pd-complexes $5 a$ and $5 b$

The Heck reaction of aryl bromides with styrene in the presence of catalysts 5a and 5b (Table 1). General Procedure

${ }^{1} \mathrm{H}$ - and ${ }^{13} \mathrm{C}$-NMR data of isolated pure products $\quad$ of the Heck reactions $\quad \mathrm{S} 4$

${ }^{1}$ H-NMR Spectrum of 5 a

${ }^{13} \mathrm{C}-\mathrm{NMR}$ Spectrum of $5 a$

${ }^{1}$ H-NMR Spectrum of 5 a S8

${ }^{13}$ C-NMR Spectrum of $5 a$ S9

References 


\section{SUPPORTING INFORMATION}

General. Unless otherwise noted, all reactions were performed under an atmosphere of dry Ar in ovendried glassware and anhydrous solvents. All aryl halides (Acros, Aldrich, Fisher, Lancaster), olefins (Aldrich), $N, N$-dimethylacetamide (anhydrous; Sure-Seal, Aldrich) were used as received from commercial sources. Triethylamine (Fisher) was distilled under argon from sodium. Potassium acetate (Aldrich) was ground into a fine powder and stored in a drying oven. $N$-tert-butyl- $\alpha$ phenylnitrone (4a) was purchased from Aldrich and used as received. N-tert-Butyl- $\alpha-(2,4-$ dimethoxyphenyl)nitrone $\quad(\mathbf{4 b})^{1} \quad$ was prepared from the condensation of 2,4dimethoxybenzaldehyde (Aldrich) and $N$-tert-butyl hydroxylamine acetate (Aldrich) in the presence of triethylamine in $\mathrm{CH}_{2} \mathrm{Cl}_{2}$ at rt . Flash chromatography was performed on silica gel 60 (230-400 mesh). Unless otherwise noted, NMR spectra were acquired at $25{ }^{\circ} \mathrm{C}$ in $\mathrm{CDCl}_{3}$ at 500 $\mathrm{MHz}$ for ${ }^{1} \mathrm{H}$ and $125 \mathrm{MHz}$ for ${ }^{13} \mathrm{C}$.

Synthesis of Pd-complexes 5a and 5b. An oven-dried Schlenk flask was charged with $\mathrm{Pd}(\mathrm{OAc})_{2}$ (112.5 mg, $\left.0.50 \mathrm{mmol}\right)$ and nitrone 4a (88.6 mg, $\left.0.50 \mathrm{mmol}\right)$ under argon. Glacial acetic acid (2 mL) was then added and the reaction mixture was heated to $90{ }^{\circ} \mathrm{C}$ (bath temperature) for $1 \mathrm{~h}$. After cooling to rt, the solvent was removed under vacuum. The crude reaction product was purified by chromatography on silica gel (EtOAc/ $\mathrm{CH}_{2} \mathrm{Cl}_{2}$ 2:3). As a substantial amount of catalyst gets stuck on the column, the column is flushed with neat ethanol, the ethanol is removed under vacuum and the recovered catalyst is purified again with silica chromatography using neat EtOAc to give $141 \mathrm{mg}$ (82\%) of the light yellow 5a as a 10:1 diastereomeric mixture: m.p. $213{ }^{\circ} \mathrm{C}$ (dec.). ${ }^{1} \mathrm{H}$ NMR (500 MHz, $\mathrm{CDCl}_{3}$ ): major isomer: $\delta 7.51$ (d, 2 H, $J=7.79 \mathrm{~Hz}$ ), 7.45 (s, 2 H), 7.08 (t, 2 H, $J=7.38 \mathrm{~Hz}$ ), 7.02 (t, 2 H, J = 7.17 Hz), 6.95 (d, $2 \mathrm{H}, J=7.35 \mathrm{~Hz}), 2.12(\mathrm{~s}, 6 \mathrm{H}), 1.18(\mathrm{~s}, 18 \mathrm{H})$; minor isomer; $\delta 7.58(\mathrm{~s}, 2 \mathrm{H}), 6.91(\mathrm{t}, 2 \mathrm{H}, J=$ $7.18 \mathrm{~Hz}$ ), 6.85 (d, $2 \mathrm{H}, J=7.41 \mathrm{~Hz}), 6.79$ (t, $2 \mathrm{H}, J=7.42 \mathrm{~Hz}), 6.73$ (d, $2 \mathrm{H}, J=7.94 \mathrm{~Hz}), 2.15$ (s, $3 \mathrm{H}), 2.01$ (s, $3 \mathrm{H}), 1.51$ (s, $18 \mathrm{H})$. NMR (125 MHz, $\mathrm{CDCl}_{3}$ ), major isomer: $\delta$ 180.7, 138.5, 138.1, 134.5, 128.8, 128.0, 125.1, 123.9, 69.4, 28.0, 24.2; minor isomer: 182.6, 179.9, 140.4, 135.3, 128.4, 128.3, 125.7, 123.7, 69.3, 28.3, 23.9. (One aromatic carbon and one $\mathrm{CH}_{3}$ are missing due to overlap with these of the major isomer). Anal. Calcd for $\mathrm{C}_{26} \mathrm{H}_{34} \mathrm{~N}_{2} \mathrm{O}_{6} \mathrm{Pd}_{2}$ : $\mathrm{C}$, 45.69; H, 5.01; N, 4.10. Found: C, 45.37; H, 5.08; N, 3.91. 


\section{SUPPORTING INFORMATION}

Pd complexe $5 \mathbf{b}$ was synthesized under identical conditions using $N$-tert-Butyl- $\alpha-(2,4$ dimethoxyphenyl)nitrone $\mathbf{4 b}$ (237 mg, $1.0 \mathrm{mmol}$ ) and $\mathrm{Pd}(\mathrm{OAc})_{2}(224.5 \mathrm{mg}, 1.0 \mathrm{mmol}$ ), and was purified to give $265 \mathrm{mg}$ (66\%) of the light yellow $5 \mathbf{b}$ as a 5:1 diastereomeric mixture: m.p. 222 ${ }^{\circ} \mathrm{C}$ (dec.). ${ }^{1} \mathrm{H}$ NMR (500 MHz, $\mathrm{CDCl}_{3}$ ), major isomer: $\delta 8.02$ (s, $2 \mathrm{H}$ ), 7.28 (s, $2 \mathrm{H}$ ), 6.69 (d, $2 \mathrm{H}$, $J=1.84 \mathrm{~Hz}$ ), 6.11 (d, 2 H, $J=2.10 \mathrm{~Hz}$ ), 3.804 (s, 6 H), 3.797 (s, 6 H), 2.08 (s, 6 H), 1.16 (s, 18 H); minor isomer: 8.08 (s, $2 \mathrm{H}), 6.06$ (d, $2 \mathrm{H}, J=1.96 \mathrm{~Hz}), 6.00(\mathrm{~d}, 2 \mathrm{H}, J=2.10 \mathrm{~Hz}$ ), 3.75 (s, 6 H), 3.53 (s, $6 \mathrm{H}), 2.11$ (s, $3 \mathrm{H}), 2.99$ (s, 3H), 1.49 (s, $18 \mathrm{H}) .{ }^{13} \mathrm{C}$ NMR (125 MHz, $\mathrm{CDCl}_{3}$ ), major isomer: $\delta$ 180.4, 159.1, 156.8, 143.3, 132.7, 108.9, 108.7, 95.4, 68.5, 55.4, 55.2, 27.9, 24.2; minor isomer: $\delta 182.3,179.9,159.4,156.7,144.1,135.2,109.6,95.2,68.4,55.3,54.9,28.3$, 24.3, 23.6. (One aromatic carbon is missing due to overlap with these of the major isomer). Anal. Calcd for $\mathrm{C}_{30} \mathrm{H}_{42} \mathrm{~N}_{2} \mathrm{O}_{10} \mathrm{Pd}_{2}$ : C, 44.84; H, 5.27; N, 3.49. Found: C, 44.73; H, 5.04; N, 3.29. Crystals suitable for X-ray crystallographic analysis were obtained from a concentrated solution of $5 \mathbf{b}$ in $\mathrm{C}_{6} \mathrm{D}_{6}$.

The Heck reaction of aryl bromides with styrene in the presence of catalysts $5 a$ and $5 \mathbf{b}$ (Table 1). General Procedure: An oven-dried Schlenk flask was charged under argon with bromobenzene (527 $\mu \mathrm{L}, 784 \mathrm{mg} 5.0 \mathrm{mmol}$ ), styrene ( $690 \mu \mathrm{L}, 648 \mathrm{mg}, 6.0 \mathrm{mmol}$ ), anhydrous KOAc (589 mg, $6.0 \mathrm{mmol}$ ) and DMA (5.0 mL). A solution of catalyst 5a in DMA (4.48 x $10^{-4}$ M, $90 \mu \mathrm{L}, 8.0 \times 10^{-4} \mathrm{~mol} \%$ ) was then added via syringe. The flask was sealed and placed in a $140{ }^{\circ} \mathrm{C}$ oil bath controlled by a J-KEMP temperature controller and stirred for $24 \mathrm{~h}$. After cooling to rt, the reaction mixture was poured into water $(200 \mathrm{~mL})$ and extracted with EtOAc (3 x $50 \mathrm{~mL}$ ). The combined organic extracts were washed with brine, dried with anhydrous $\mathrm{Na}_{2} \mathrm{SO}_{4}$, and concentrated. Purification by flash chromatography on silica gel (hexanes $/ \mathrm{CH}_{2} \mathrm{Cl}_{2}$ 1:1) gave trans-stilbene along with a trace amount of its regioisomer (790 mg, 87\%). In another parallel run under identical conditions, the reaction gave $778 \mathrm{mg}$ (86\%) of the products after workup and purification. 


\section{SUPPORTING INFORMATION}

\section{${ }^{1} \mathrm{H}$ - and ${ }^{13} \mathrm{C}-\mathrm{NMR}$ data of isolated pure products of the Heck reactions}

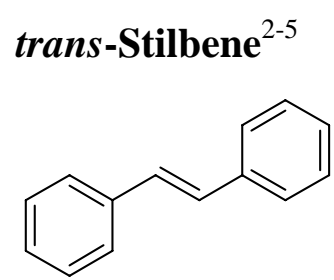

${ }^{1} \mathrm{H}$ NMR (500 MHz, $\mathrm{CDCl}_{3}$ ): $\delta 7.66$ (d, $4 \mathrm{H}, J=7.8 \mathrm{~Hz}$ ), 7.51 (t, $\left.4 \mathrm{H}, J=7.6 \mathrm{~Hz}\right), 7.41$ (t, $2 \mathrm{H}, J$ = 7.3 Hz), 7.27 (s, $2 \mathrm{H}) .{ }^{13} \mathrm{C}$ NMR (125 MHz, $\left.\mathrm{CDCl}_{3}\right): \delta 137.51,128.88,128.84,127.77,126.70$.

\section{4-Methoxy-trans-stilbene ${ }^{2-4}$}<smiles>COc1ccc(/C=C/c2ccccc2)cc1</smiles>

${ }^{1} \mathrm{H}$ NMR (500 MHz, $\mathrm{CDCl}_{3}$ ): $\delta 7.54$ (d, $\left.2 \mathrm{H}, \mathrm{d}, J=7.8 \mathrm{~Hz}\right), 7.50$ (d, $\left.2 \mathrm{H}, J=8.7 \mathrm{~Hz}\right), 7.39$ (t, 2 H, $J=7.6 \mathrm{~Hz}$ ), 7.28 (t, $1 \mathrm{H}, J=7.3 \mathrm{~Hz}), 7.12$ (d, $1 \mathrm{H}, J=16.3 \mathrm{~Hz}), 7.02$ (d, $1 \mathrm{H}, J=16.3 \mathrm{~Hz}$ ), 6.95 (d, $2 \mathrm{H}, J=8.7 \mathrm{~Hz}$ ), 3.87 (s, $3 \mathrm{H}) .{ }^{13} \mathrm{C} \mathrm{NMR}\left(125 \mathrm{MHz}, \mathrm{CDCl}_{3}\right): \delta 159.37,137.71,130.21$, 128.67, 128.21, 127.75, 127.23, 126.68, 126.29, 114.19, 55.34.

\section{4-Methyl-trans-stilbene ${ }^{3}$}<smiles>Cc1ccc(/C=C/c2ccccc2)cc1</smiles>

${ }^{1} \mathrm{H}$ NMR (500 MHz, $\mathrm{CDCl}_{3}$ ): $\delta 7.69$ (d, $2 \mathrm{H}, J=7.7 \mathrm{~Hz}$ ), 7.61 (d, $\left.2 \mathrm{H}, J=8.1 \mathrm{~Hz}\right), 7.54$ (t, $2 \mathrm{H}, J$ = 7.6 Hz), 7.44 (t, $1 \mathrm{H}, J=7.3 \mathrm{~Hz}$ ), 7.36 (d, $2 \mathrm{H}, J=7.9 \mathrm{~Hz}), 7.30$ (d, $1 \mathrm{H}, J=16.3 \mathrm{~Hz}), 7.26$ (d, $1 \mathrm{H}, J=16.3 \mathrm{~Hz}$ ), 2.55 (s, $3 \mathrm{H}) .{ }^{13} \mathrm{C} \mathrm{NMR}\left(125 \mathrm{MHz}, \mathrm{CDCl}_{3}\right): \delta 137.85,137.66,134.89,129.70$, 128.92, 128.02, 127.66, 126.79, 126.75, 21.51 (One aromatic carbon is missing due to overlap).

2-Methyl-trans-stilbene $e^{2,3,5}$ 


\section{SUPPORTING INFORMATION}<smiles>Cc1ccccc1/C=C/c1ccccc1</smiles>

${ }^{1} \mathrm{H}$ NMR (500 MHz, $\mathrm{CDCl}_{3}$ ): $\delta 7.88$ (d, $1 \mathrm{H}, J=7.2 \mathrm{~Hz}$ ), 7.80 (d, $2 \mathrm{H}, J=7.6 \mathrm{~Hz}$ ), 7.61-7.65 (m, $3 \mathrm{H}), 7.44-7.55$ (m, $4 \mathrm{H}), 7.29$ (d, $1 \mathrm{H}, J=16.1 \mathrm{~Hz}$ ), 2.70 (s, $3 \mathrm{H}) .{ }^{13} \mathrm{C}$ NMR (125 MHz, $\mathrm{CDCl}_{3}$ ): $\delta$ 138.24, 136.90, 136.20, 130.94, 130.51, 129.17, 128.06, 127.12, 127.03, 126.75, 125.97, 20.35 (One aromatic carbon is missing due to overlap).

\section{4-Nitro-trans-stilbene $e^{4,5}$}<smiles>O=[N+]([O-])c1ccc(/C=C/c2ccccc2)cc1</smiles>

${ }^{1} \mathrm{H}$ NMR (500 MHz, $\mathrm{CDCl}_{3}$ ): $\delta 8.23$ (d, $2 \mathrm{H}, J=8.8 \mathrm{~Hz}$ ), 7.64 (d, $2 \mathrm{H}, J=8.8 \mathrm{~Hz}$ ), 7.58 (d, $2 \mathrm{H}$, $J=7.4 \mathrm{~Hz}$ ), 7.43 (t, $2 \mathrm{H}, J=7.45 \mathrm{~Hz}$ ), 7.37 (t, $1 \mathrm{H}, J=7.3 \mathrm{~Hz}), 7.29$ (d, $1 \mathrm{H}, J=16.3 \mathrm{~Hz}), 7.16$ (d, $1 \mathrm{H}, J=16.3 \mathrm{~Hz}) .{ }^{13} \mathrm{C}$ NMR $\left(125 \mathrm{MHz} \mathrm{CDCl}_{3}\right): \delta 146.94,143.88,136.30,133.37,128.88$, 128.81, 127.03, 126.84, 126.36, 124.08. 


\section{SUPPORTING INFORMATION}

\section{${ }^{1}$ H-NMR Spectrum of $5 a$}

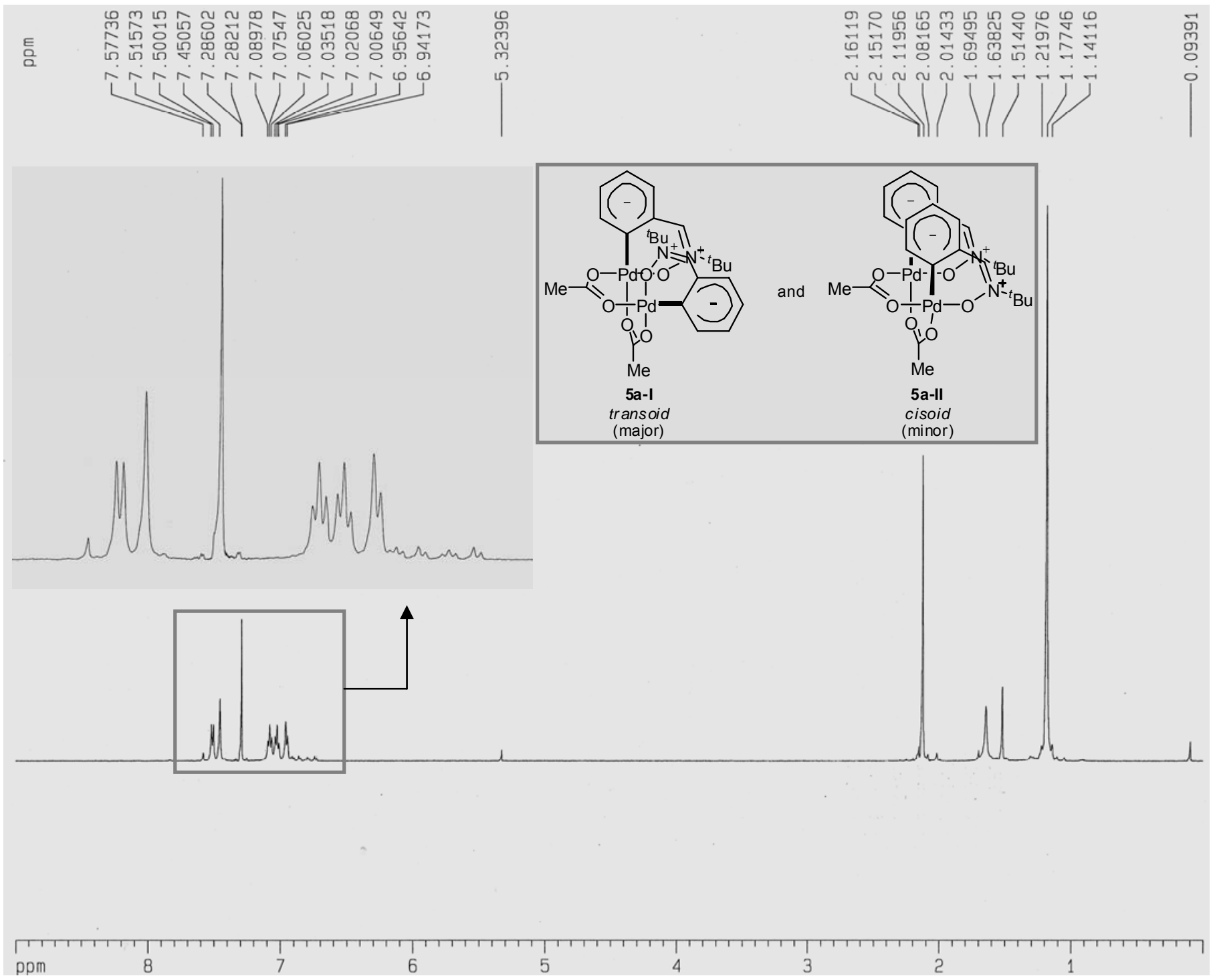




\section{SUPPORTING INFORMATION}

\section{${ }^{13}$ C-NMR Spectrum of 5a}

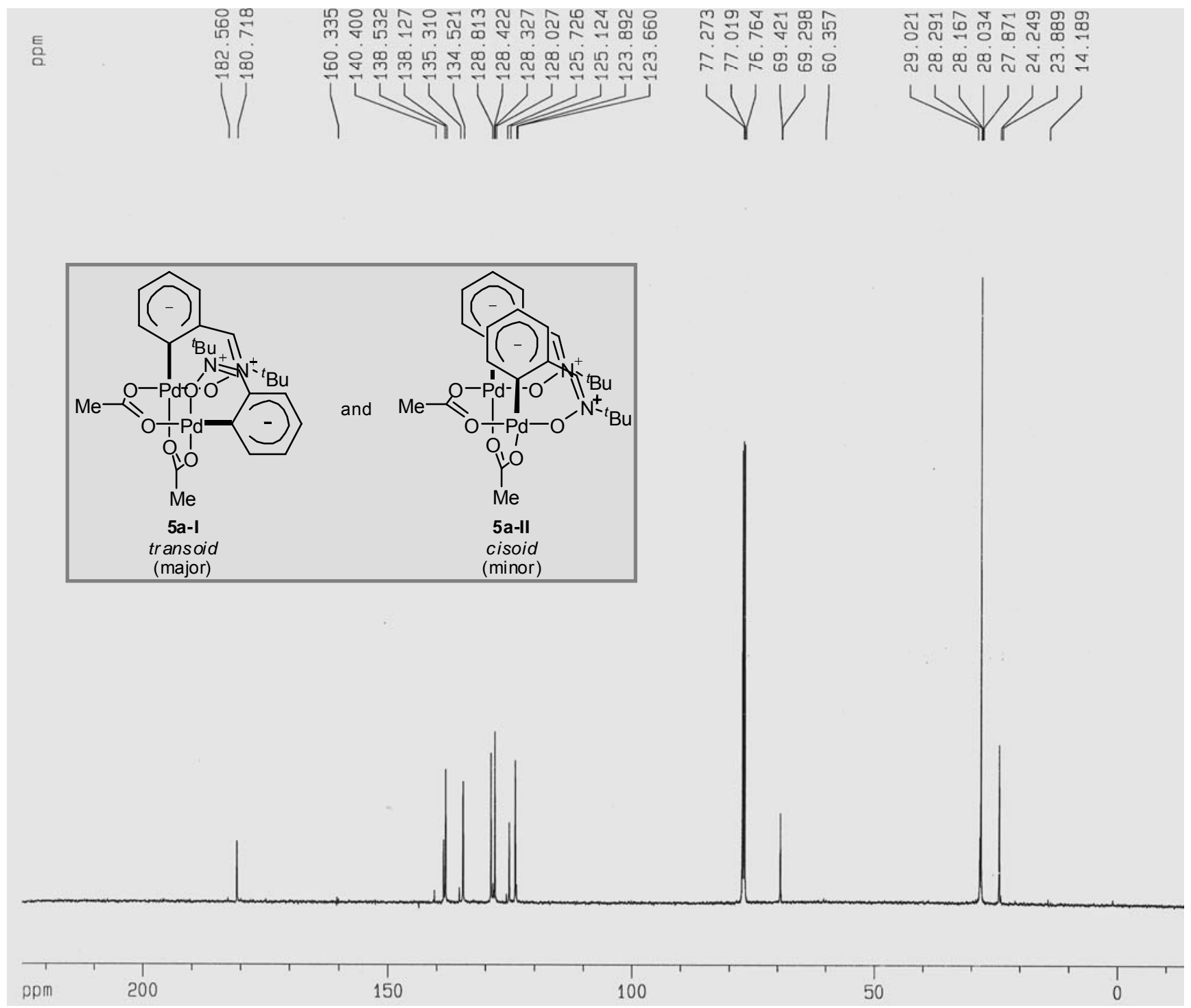




\section{SUPPORTING INFORMATION}

\section{${ }^{1}$ H-NMR Spectrum of $5 b$}

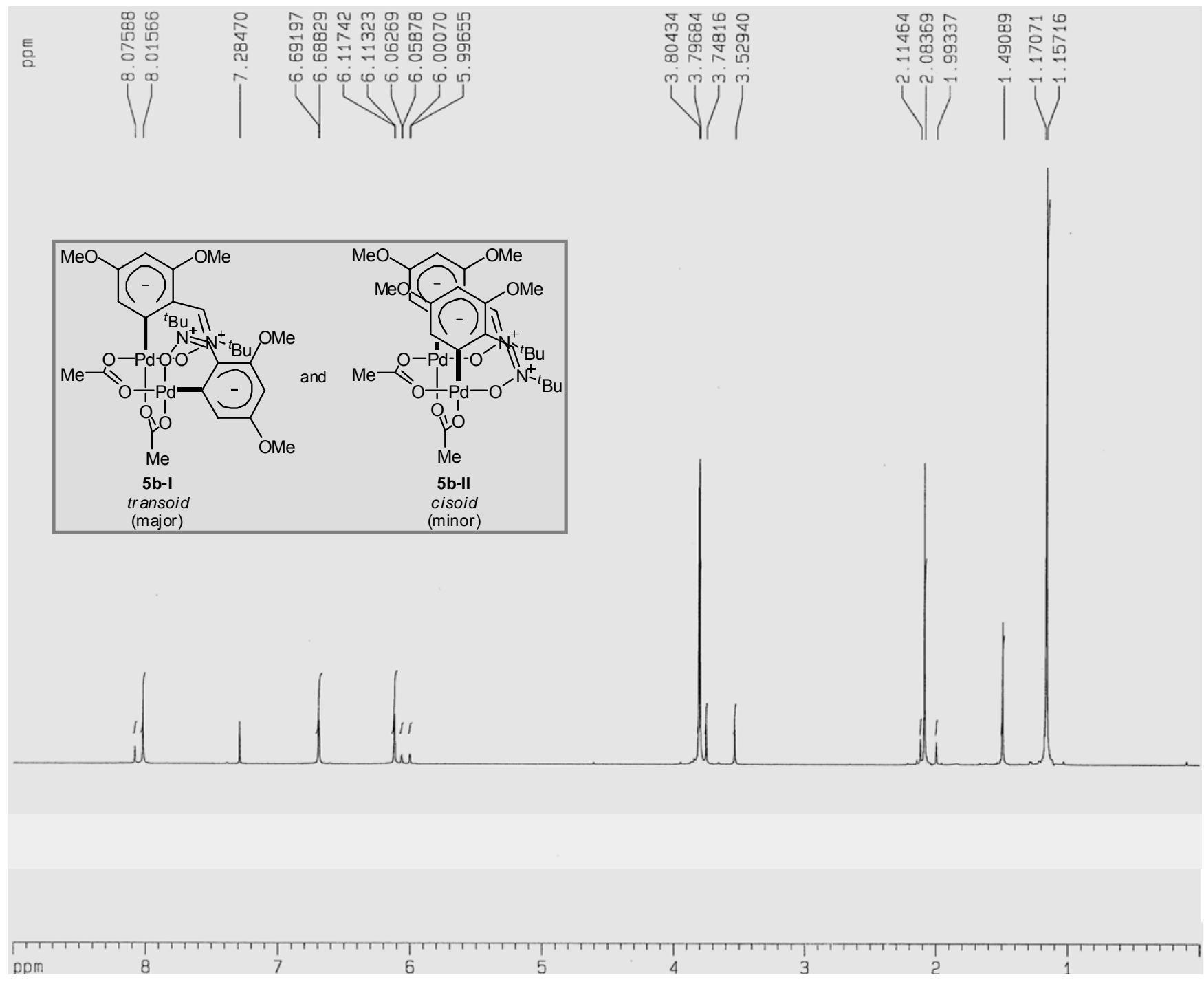




\section{SUPPORTING INFORMATION}

${ }^{13}$ C-NMR Spectrum of $5 b$

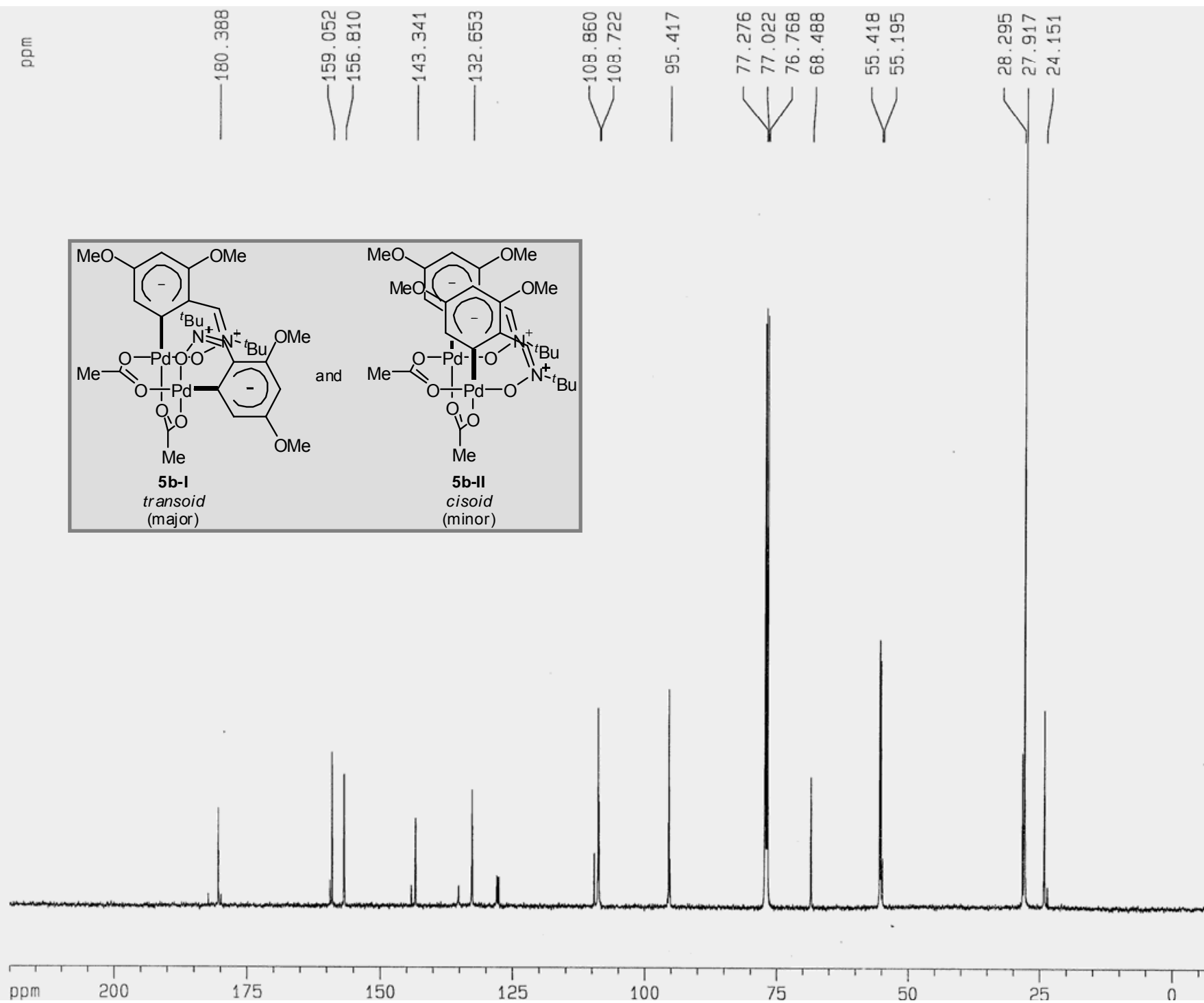




\section{SUPPORTING INFORMATION}

\section{References}

1. (a) Zinner, G; Eghtessad, E. Archiv der Pharmazie 1979, 312, 907. (b) Kloc, K.; Kubicz, E.; Mlochowski, J.; Syper, L. Synthesis 1987, 1084.

2. Littke, A. F.; Fu, G. C. J. Org. Chem. 1999; 64, 10.

3. Yao, Q.; Kinney, E. P.; Zheng, C. Org. Lett. 2004, 6, 2997.

4. Xiong, Z.; Wang, N.; Dai, M.; Li, A.; Chen, J.; Yang. Z. Org. Lett. 2004; 6, 3337.

5. Masllorens, J.; Moreno-Manas, M.; Pla-Quintana, A.; Roglans, A. Org. Lett. 2003; 5, 1559. 